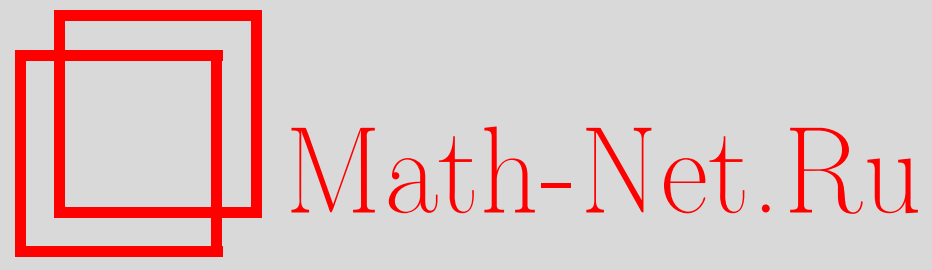

Е. А. Козлова, Задача о полном успокоении для одного класса систем гиперболических уравнений второго порядка, Вестн. Сам. гос. техн. ун-та. Сер. Физ.-мат. науки, 2012, выпуск 3(), 47-52

DOI: https://doi.org/10.14498/vsgtu1072

Использование Общероссийского математического портала Math-Net.Ru подразумевает, что вы прочитали и согласны с пользовательским соглашением

http: //www . mathnet.ru/rus/agreement

Параметры загрузки:

IP : 3.85 .73 .92

26 апреля 2023 г., 03:24:28

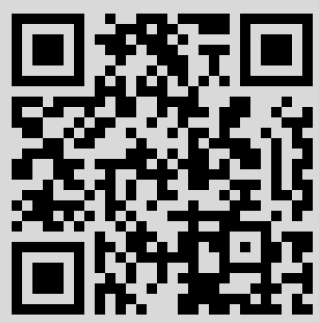


УДК 517.956.3

\section{ЗАДАЧА О ПОЛНОМ УСПОКОЕНИИ ДЛЯ ОДНОГО КЛАССА СИСТЕМ ГИПЕРБОЛИЧЕСКИХ УРАВНЕНИЙ ВТОРОГО ПОРЯДКА}

\section{E. А. Козлова}

Самарский государственный технический университет, 443100, Россия, Самара, ул. Молодогвардейская, 244.

E-mail: leni2006@mail.ru

Рассмотрен частный случай задачи управления - задача о полном успокоении для системы гиперболических уравнений, содержащей смешанную производную. Для различных случаев совершён переход от исходной системы уравнений $\kappa$ системе треугольного или диагонального вида, допускающей расщепление уравнений. Соответствующим образом преобразованы начальные и финальные данные. С помощъю решения задачи Коши для однородного или неоднородного уравнений построены две компоненты векторов граничного управления, дающие после обратной замены искомые управляющие бункции.

Ключевые слова: граничное управление, задача о полном успокоении, задача Коши, система гиперболических уравнений, смешанная производная.

\section{Рассмотрим систему уравнений}

$$
u_{t t}+2 B u_{x t}+C u_{x x}=0,
$$

где $B, C$ - постоянные коммутирующие матрицы размерности $2 \times 2, u(x, t)-$ двумерная вектор-функция.

Сделаем следующие предположения относительно собственных значений матриц $B$ и $C$ :

1) $\lambda_{1 B}=\lambda_{2 B}=\lambda_{B}$

2) $\lambda_{B}^{2}-\lambda_{1 C}>0, \lambda_{B}^{2}-\lambda_{2 C}>0$ (это условие обеспечивает гиперболичность [1] системы (1)).

Для системы $(1)$ в прямоугольнике $Q=[0, l] \times[0, T]$ рассмотрим частный случай задачи управления - задачу о полном успокоении с начальными

$$
u(x, 0)=\varphi(x), \quad u_{t}(x, 0)=\psi(x), \quad 0 \leqslant x \leqslant l
$$

и финальными

$$
u(x, T)=0, \quad u_{t}(x, T)=0, \quad 0 \leqslant x \leqslant l
$$

условиями, при этом требуется построить функции граничного управления

$$
\mu(t)=u(0, t), \quad \nu(t)=u(l, t), \quad 0 \leqslant t \leqslant T .
$$

Здесь $\varphi(x), \psi(x), \mu(t), \nu(t)$ - двумерные вектор-функции.

Матрица $B$ имеет одно собственное значение $\lambda_{B}$ алгебраической кратности 2, геометрическая кратность которого может быть равна 2 или 1 [2].

Рассмотрим первый случай, когда нормальная жорданова форма матрицы $B$ имеет вид $J_{B}=\left(\begin{array}{cc}\lambda_{B} & 0 \\ 0 & \lambda_{B}\end{array}\right)=\lambda_{B} E$. Очевидно, что любая матрица,

Елена Александровна Козлова, аспирант, каф. прикладной математики и информатики. 
подобная $B$, будет диагональной. Выберем матрицу перехода $S(\operatorname{det} S \neq 0)$ так, чтобы она приводила $C$ к нормальной жордановой форме:

$$
J_{C}=S^{-1} C S \text {. }
$$

После замены $u=S w$ и умножения слева на $S^{-1}$ система (1) примет вид

$$
w_{t t}+2 J_{B} w_{x t}+J_{C} w_{x x}=0,
$$

начальные и финальные условия сведутся к

$$
w(x, 0)=S^{-1} \varphi(x)=\tilde{\varphi}(x), \quad w_{t}(x, 0)=S^{-1} \psi(x)=\tilde{\psi}(x), \quad 0 \leqslant x \leqslant l,
$$

и

$$
w(x, T)=0, \quad w_{t}(x, T)=0, \quad 0 \leqslant x \leqslant l,
$$

соответственно, а граничные управления - к

$$
\tilde{\mu}(t)=w(0, t)=S^{-1} \mu(t), \quad \tilde{\nu}(t)=w(l, t)=S^{-1} \nu(t), \quad 0 \leqslant t \leqslant T .
$$

Если матрица $C$ - простая, то $J_{C}$ является диагональной, иначе - треугольной.

Выделим следующие варианты:

а) $J_{C}=\left(\begin{array}{cc}\lambda_{1 C} & 0 \\ 0 & \lambda_{2 C}\end{array}\right)$;

б) матрица $C$ не является простой, $\lambda_{1 C}=\lambda_{2 C}=\lambda_{C}, J_{C}=\left(\begin{array}{cc}\lambda_{C} & 0 \\ 1 & \lambda_{C}\end{array}\right)$.

Рассмотрим вариант а). В этом случае система распадается на два уравнения, для $k$-той компоненты вектор-функции $w(x, t)(k=1,2)$ из $(2)-(4)$ получаем задачу управления в области $Q$, состоящую из уравнения

$$
\left(w_{k}\right)_{t t}+2 \lambda_{B}\left(w_{k}\right)_{x t}+\lambda_{k C}\left(w_{k}\right)_{x x}=0
$$

начальных условий

$$
w_{k}(x, 0)=\tilde{\varphi}_{k}(x), \quad\left(w_{k}\right)_{t}(x, 0)=\tilde{\psi}_{k}(x), \quad 0 \leqslant x \leqslant l
$$

и нулевых финальных условий.

Введём четыре действительных коэффициента:

$$
\begin{aligned}
& p_{11}=\lambda_{B}-\sqrt{\lambda_{B}^{2}-\lambda_{1 C}}, \quad p_{21}=\lambda_{B}+\sqrt{\lambda_{B}^{2}-\lambda_{1 C}}, \\
& p_{12}=\lambda_{B}-\sqrt{\lambda_{B}^{2}-\lambda_{2 C}}, \quad p_{22}=\lambda_{B}+\sqrt{\lambda_{B}^{2}-\lambda_{2 C}} .
\end{aligned}
$$

Построение решения задачи успокоения для $w_{k}$ при $p_{2 k}>-p_{1 k}>0$ (аналогично $p_{1 k}<-p_{2 k}<0$ ) было описано в [3], при $p_{2 k}>p_{1 k}>0$ (или $\left.p_{1 k}<p_{2 k}<0\right)-$ в [4].

Пусть $p_{2 k}>p_{1 k}>0, \gamma_{k}=\left(p_{2 k}-p_{1 k}\right)^{-1}$ и время управления достаточно мало: $T \leqslant l / p_{2 k}, k=1,2$ (остальные случаи рассматриваются аналогично). Прямые $t=0, t=T$, на которых заданы начальные и финальные данные, и 
характеристики $x-p_{1 k} t=-p_{1 k} T, x-p_{2 k} t=l-p_{2 k} T, x-p_{2 k} t=0, x-p_{1 k} t=l$ образуют две треугольные области $\Delta_{1 k}=\left\{p_{2 k} t \leqslant x \leqslant p_{1 k} t+l, 0 \leqslant t \leqslant l \gamma_{k}\right\}$ и $\Delta_{2 k}=\left\{-p_{1 k}(T-t) \leqslant x \leqslant l-p_{2 k}(T-t), T-l \gamma_{k} \leqslant t \leqslant T\right\}$. Чтобы построить решение задачи управления, нужно решить две задачи Коши в $\Delta_{1 k}, \Delta_{2 k}$. Поскольку время управления мало, эти области имеют общую часть, в которой решения задач должны совпадать.

Тогда, если выполняются условия при $0 \leqslant x \leqslant l-p_{2 k} T$ :

$$
\tilde{\varphi}_{k}(x)=0, \quad \tilde{\psi}_{k}(x)=0
$$

и при $l-p_{2 k} T \leqslant x \leqslant l-p_{1 k} T$ :

$$
p_{2 k} \tilde{\varphi}_{k}(x)+\int_{l-p_{2 k} T}^{x} \tilde{\psi}_{k}(z) d z=0,
$$

то граничные управления имеют вид:

$$
\begin{gathered}
\tilde{\mu}_{k}(t)=0 \\
\tilde{\nu}_{k}(t)=\gamma_{k}\left(p_{2 k} \tilde{\varphi}_{k}\left(l-p_{1 k} t\right)-p_{1 k} \tilde{\varphi}_{k}\left(l-p_{2 k} t\right)+\int_{l-p_{2 k} t}^{l-p_{1 k} t} \tilde{\psi}_{k}(z) d z\right) .
\end{gathered}
$$

Следовательно, определены все компоненты функций $\tilde{\mu}(t), \tilde{\nu}(t)$ и

$$
\mu(t)=S \tilde{\mu}(t), \quad \nu(t)=S \tilde{\nu}(t) .
$$

Рассмотрим вариант б). В этом случае получаем систему вида

$$
\left\{\begin{array}{l}
\left(w_{1}\right)_{t t}+2 \lambda_{B}\left(w_{1}\right)_{x t}+\lambda_{C}\left(w_{1}\right)_{x x}=0 \\
\left(w_{2}\right)_{t t}+2 \lambda_{B}\left(w_{2}\right)_{x t}+\lambda_{C}\left(w_{2}\right)_{x x}=-\left(w_{1}\right)_{x x}
\end{array}\right.
$$

с начальными условиями (3) и нулевыми финальными условиями, $p_{11}=p_{12}=$ $=p_{1}, p_{21}=p_{22}=p_{2}, \gamma_{1}=\gamma_{2}=\gamma$.

Решение задачи управления для $w_{1}$ имеет тот же вид, что и в предыдущем случае. Функции $\tilde{\mu}_{1}, \tilde{\nu}_{1}$ определяются формулами $(7),(8)$. Второе уравнение системы является неоднородным, его правая часть зависит от $w_{1}$, то есть известна. Два уравнения системы имеют одинаковые характеристики, поэтому области построения решений совпадают $\left(\Delta_{11}=\Delta_{12}, \Delta_{21}=\Delta_{22}\right)$. Следовательно, находя в этих областях $w_{2}$ в виде суммы решения однородного уравнения, удовлетворяющего неоднородным условиям, и частного решения соответствующего неоднородного уравнения с однородными условиями, получим искомые функции $\tilde{\mu}_{2}, \tilde{\nu}_{2}$ :

$$
\begin{gathered}
\tilde{\mu}_{2}(t)=\gamma^{3}\left(p_{2} \tilde{\varphi}_{1}\left(-p_{1} t\right)-p_{1} \tilde{\varphi}_{1}\left(-p_{2} t\right)+p_{1} \tilde{\varphi}_{1}\left(-p_{1} t-\gamma^{-1} T\right)-p_{2} \tilde{\varphi}_{1}\left(-p_{2} t+\gamma^{-1} T\right)\right)+ \\
\quad+\gamma^{3} \int_{-p_{2} t}^{-p_{1} t-\gamma^{-1} T}\left(\tilde{\psi}_{1}\left(z+\gamma^{-1} T\right)+\tilde{\psi}_{1}(z)\right) d z+ \\
+\gamma^{2}(T-t)\left(p_{2} \tilde{\varphi}_{1}^{\prime}\left(-p_{1} t\right)+p_{1} \tilde{\varphi}_{1}^{\prime}\left(-p_{2} t\right)+\tilde{\psi}_{1}\left(-p_{1} t\right)+\tilde{\psi}_{1}\left(-p_{2} t\right)\right),
\end{gathered}
$$




$$
\begin{aligned}
\tilde{\nu}_{2}(t)= & \gamma\left(p_{2} \tilde{\varphi}_{2}\left(l-p_{1} t\right)-p_{1} \tilde{\varphi}_{2}\left(l-p_{2} t\right)+\int_{l-p_{2} t}^{l-p_{1} t} \tilde{\psi}_{2}(z) d z\right)+ \\
& +\gamma^{3}\left(p_{1}+p_{2}\right)\left(\tilde{\varphi}_{1}\left(l-p_{1} t\right)-\tilde{\varphi}_{1}\left(l-p_{2} t\right)\right)+2 \gamma^{3} \int_{l-p_{2} t}^{l-p_{1} t} \tilde{\psi}_{1}(z) d z- \\
& \quad-\gamma^{2} t\left(p_{2} \tilde{\varphi}_{1}^{\prime}\left(l-p_{1} t\right)+p_{1} \tilde{\varphi}_{1}^{\prime}\left(l-p_{2} t\right)+\tilde{\psi}_{1}\left(l-p_{1} t\right)+\tilde{\psi}_{1}\left(l-p_{2} t\right)\right) .
\end{aligned}
$$

При этом должны выполняться условия $(5),(6)$ при $k=1$, а также условия при $0 \leqslant x \leqslant l-p_{2 k} T$ :

$$
\tilde{\varphi}_{2}(x)=0, \quad \tilde{\psi}_{2}(x)=0
$$

и при $l-p_{2 k} T \leqslant x \leqslant l-p_{1 k} T$ :

$$
\begin{aligned}
\gamma^{2}\left(p_{1} \tilde{\varphi}_{1}(x)-p_{1} \tilde{\varphi}_{1}\left(x-\gamma^{-1} T\right)+\int_{l-\gamma^{-1} T}^{l-p_{2} T} \tilde{\psi}_{1}(z) d z\right)+ & \\
& +p_{2} \tilde{\varphi}_{2}(x)+\int_{l-p_{2} T}^{x} \tilde{\psi}_{2}(z) d z=0 .
\end{aligned}
$$

Замена (9) заканчивает решение.

Теперь предположим, что нормальная жорданова форма матрицы $B$ имеет вид $J_{B}=\left(\begin{array}{cc}\lambda_{B} & 0 \\ 1 & \lambda_{B}\end{array}\right)$. Матрица перехода $S(\operatorname{det} S \neq 0)$ должна приводить $B$ к нормальной жордановой форме: $J_{B}=S^{-1} B S$. Обозначим $J_{C}=S^{-1} C S$. $J_{B} J_{C}=J_{C} J_{B}$, так как $B C=C B$. Известен общий вид матрицы, коммутирующей с $J_{B}[2]: J_{C}=\left(\begin{array}{cc}\lambda_{C} & 0 \\ \alpha & \lambda_{C}\end{array}\right)$. Здесь $\alpha$-число, которое в зависимости от структуры $C$ может быть равно нулю или отлично от нуля.

Тогда исследуемая система перейдет в систему

$$
\left\{\begin{array}{l}
\left(w_{1}\right)_{t t}+2 \lambda_{B}\left(w_{1}\right)_{x t}+\lambda_{C}\left(w_{1}\right)_{x x}=0 \\
\left(w_{2}\right)_{t t}+2 \lambda_{B}\left(w_{2}\right)_{x t}+\lambda_{C}\left(w_{2}\right)_{x x}=-2\left(w_{1}\right)_{x t}-\alpha\left(w_{1}\right)_{x x}
\end{array}\right.
$$

Начальные и финальные условия - такие же, как и в предыдущем случае. Разделяем систему на два уравнения (однородное и неоднородное) и строим решения получившихся задач, как и ранее, что и приводит к искомым функциям граничного управления. Поскольку частные решения для правой части, содержащей $\left(w_{1}\right)_{x x}$, были построены выше, ограничимся случаем $\alpha=$ $=0$. Итак, построены управления в виде:

$$
\begin{gathered}
\tilde{\mu}_{2}(t)=-2 \gamma^{3} p_{1} p_{2}\left(\tilde{\varphi}_{1}\left(-p_{1} t\right)-\tilde{\varphi}_{1}\left(-p_{2} t\right)+\tilde{\varphi}_{1}\left(-p_{1} t-\gamma^{-1} T\right)-\tilde{\varphi}_{1}\left(-p_{2} t+\gamma^{-1} T\right)\right)- \\
-2 \gamma^{3} \int_{-p_{2} t}^{-p_{1} t-\gamma^{-1} T}\left(p_{1} \tilde{\psi}_{1}\left(z+\gamma^{-1} T\right)+p_{2} \tilde{\psi}_{1}(z)\right) d z- \\
-2 \gamma^{2}(T-t)\left(p_{1} p_{2} \tilde{\varphi}_{1}^{\prime}\left(-p_{1} t\right)+p_{1} p_{2} \tilde{\varphi}_{1}^{\prime}\left(-p_{2} t\right)+p_{1} \tilde{\psi}_{1}\left(-p_{1} t\right)+p_{2} \tilde{\psi}_{1}\left(-p_{2} t\right)\right)
\end{gathered}
$$




$$
\begin{aligned}
& \tilde{\nu}_{2}(t)=\gamma\left(p_{2} \tilde{\varphi}_{2}\left(l-p_{1} t\right)-p_{1} \tilde{\varphi}_{2}\left(l-p_{2} t\right)+\int_{l-p_{2} t}^{l-p_{1} t} \tilde{\psi}_{2}(z) d z\right)- \\
& \quad-4 \gamma^{3} p_{1} p_{2}\left(\tilde{\varphi}_{1}\left(l-p_{1} t\right)-\tilde{\varphi}_{1}\left(l-p_{2} t\right)\right)-2 \gamma^{3}\left(p_{1}+p_{2}\right) \int_{l-p_{2} t}^{l-p_{1} t} \tilde{\psi}_{1}(z) d z+ \\
& +2 \gamma^{2} t\left(p_{1} p_{2} \tilde{\varphi}_{1}^{\prime}\left(l-p_{1} t\right)+p_{1} p_{2} \tilde{\varphi}_{1}^{\prime}\left(l-p_{2} t\right)+p_{1} \tilde{\psi}_{1}\left(l-p_{1} t\right)+p_{2} \tilde{\psi}_{1}\left(l-p_{2} t\right)\right) .
\end{aligned}
$$

Должны выполняться соотношения $(5),(6)(k=1)$, условия при $0 \leqslant x \leqslant$ $\leqslant l-p_{2 k} T$ :

$$
\tilde{\varphi}_{2}(x)=0, \quad \tilde{\psi}_{2}(x)=0,
$$

и при $l-p_{2 k} T \leqslant x \leqslant l-p_{1 k} T$ :

$$
\begin{aligned}
& 2 \gamma T\left(\tilde{\varphi}_{1}^{\prime}(x)+p_{1} \tilde{\psi}_{1}(x)\right)+2 \gamma^{2} p_{1} p_{2} \tilde{\varphi}_{1}\left(x-\gamma^{-1} T\right)+ \\
+ & 2 \gamma^{2}\left(p_{1} \int_{l-p_{2} T}^{x} \tilde{\psi}_{1}(z) d z+p_{2} \int_{x}^{x-\gamma^{-1} T} \tilde{\psi}_{1}(z) d z\right)+p_{2} \tilde{\varphi}_{2}(x)+\int_{l-p_{2} T}^{x} \tilde{\psi}_{2}(z) d z=0 .
\end{aligned}
$$

Для возвращения к функциям $\mu(t), \nu(t)$ выполняется преобразование (9).

Задачи управления для гиперболических уравнений изучались многими авторами. В данной статье использованы работы В. А. Ильина и Е. И. Моисеева $[5,6]$, исследующие управление для волнового и телеграфного уравнений, а также работы А. А. Андреева и С. В. Лексиной [7] для системы уравнений гиперболического типа. Отметим, что гиперболическое уравнение, содержащее смешанную производную, возникает при описании малых колебаний движущегося гибкого стержня $[8,9]$.

\section{БИБЛИОГРАФИЧЕСКИЙ СПИСОК}

1. Бищадзе А. В. Некоторые классы уравнений в частных производных. М.: Наука, 1981. 448 c. [Bitsadze A. V. Some classes of partial differential equations. Moscow: Nauka, 1981. 448 pp.]

2. Гантмахер Ф. Р. Теория матриц. М.: Наука, 1988. 549 с. [Gantmakher F. R. Theory of matrices. Moscow: Nauka, 1988. 549 pp.]

3. Козлова E. А. Задача о полном успокоении для гиперболического уравнения, содержащего смешанную производную// Вестн. Сам. гос. техн. ун-та. Сер. Физ.-мат. науки, 2011. № 4(25). C. 37-42. [Kozlova E. A. Damping problem for the hyperbolic equation with mixed derivative// Vestn. Samar. Gos. Tekhn. Univ. Ser. Fiz.-Mat. Nauki, 2011. Vol. 4(25). Pp. 37-42].

4. Козлова E. A. Задача управления для гиперболического уравнения в случае характеристик с угловыми коэффициентами одного знака// Becmн. Сам. гос. техн. ун-та. Cер. Физ.-мат. науки, 2012. Т. 1(26). С. 243-247. [Kozlova E. A. Control problem for the hyperbolic equation with the characteristics having the angular coefficients of the same sign // Vestn. Samar. Gos. Tekhn. Univ. Ser. Fiz.-Mat. Nauki, 2012. no.1(26). Pp. 243-247].

5. Ильин B. A. Граничное управление процессом колебаний на двух концах в терминах обобщенного решения волнового уравнения с конечной энергией // Дифферени. уравнения, 2000. Т. 36, № 11. С. 1513-1528; англ. пер.: Il'in V. A. Boundary control of oscillations on two ends in terms of the generalized solution of the wave equation with finite energy // Differ. Equ., 2000. Vol. 36, no. 11. Pp. 1659-1675.

6. Илъин B. A., Моисеев Е. И. Граничное управление на двух концах процессом, описываемым телеграфным уравнением // Докл. РAH, 2004. Т. 394, № 2. С. 154-158. [Il'in V. A., 
Moiseev E. I. Boundary control at two endpoints of a process described by the telegraph equation // Dokl. Akad. Nauk, 2004. Vol.394, no. 2. Pp. 154-158].

7. Андреев A. A., Лексина C. В. Задача граничного управления в условиях первой краевой задачи для системы гиперболического типа второго порядка // Дифферени. уравнения, 2011. T. 47, № 6. C. 843-849; англ. пер.: Andreev A. A., Leksina S. V. Boundary control problem for the first boundary value problem for a second-order system of hyperbolic type // Differ. Equ., 2011. Vol.47, no. 6. Pp. 848-854.

8. Светлицкий B. A. Механика гибких стержней и нитей. М.: Машиностроение, 1978. 224 с. [Svetlitskiy B. A. Mechanics of Flexible Rods and Threads. Moscow: Mashinostroenie, 1978. 224 pp.]

9. Скоробогатько В.Я. Исследования по качественной теории дифференциальных уравнений с частными производными. Киев: Наук. думка, 1980. 244 с. [Skorobogat'ko V. Ja. Investigation in the qualitative theory of partial differential equations. Kiev: Naukova Dumka, 1980. 244 pp.]

Поступила в редакцию $02 / \mathrm{V} / 2012$;

в окончательном варианте - 12/VII/2012.

\section{MSC: 35L51}

\section{DAMPING PROBLEM FOR THE SPECIAL CLASS OF THE SECOND ORDER HYPERBOLIC SYSTEMS}

\section{E. A. Kozlova}

Samara State Technical University,

244, Molodogvardeyskaya st., Samara, 443100, Russia.

E-mail: leni2006@mail.ru

We consider the damping problem for the hyperbolic system with mixed derivative as the special case of boundary control problem. For different cases the given system is transformed to the triangular or diagonal form, allowing separation of equations. The corresponding transformation is applied to the initial and final data. Two components of boundary control vectors are constructed by solving the Cauchy problem for homogeneous or inhomogeneous equation. The inverse transformation gives the desired control functions.

Key words: boundary control, damping problem, Cauchy problem, hyperbolic system, mixed derivative.

Original article submitted $02 / \mathrm{V} / 2012$;

revision submitted 12/VII/2012.

Elena A. Kozlova, Postgraduate Student, Dept. of Applied Mathematics \& Computer Science. 